\title{
Comprehensive Analysis of the Tumor Microenvironment in Cutaneous Melanoma associated with Immune Infiltration
}

\author{
Pan Wang ${ }^{1,}$, , Xinyu Zhang ${ }^{2,}$, , Nan Sun ${ }^{1, *}$, Zhihong Zhao ${ }^{1}$, Jie He $\mathrm{H}^{\circledR}$ \\ 1. Department of Thoracic Surgery, National Cancer Center/National Clinical Research Center for Cancer/Cancer Hospital, Chinese Academy of Medical \\ Sciences and Peking Union Medical College, Beijing 100021, China \\ 2. Department of body contouring and liposuction center, Plastic Surgery Hospital, Chinese Academy of Medical Sciences and Peking Union Medical College, \\ Beijing 100144, China \\ *These authors contributed equally to this work. \\ $\triangle$ Corresponding author: Jie He, E-mail: prof.jiehe@gmail.com. National Cancer Center/National Clinical Research Center for Cancer/Cancer Hospital, \\ Chinese Academy of Medical Sciences and Peking Union Medical College, Beijing 100021, China. Tel: 86-10-87788863. Fax numbers: +86-10-67713359. \\ () The author(s). This is an open access article distributed under the terms of the Creative Commons Attribution License (https://creativecommons.org/licenses/by/4.0/). \\ See http://ivyspring.com/terms for full terms and conditions.
}

Received: 2020.01.31; Accepted: 2020.03.27; Published: 2020.04.06

\begin{abstract}
Accumulating evidence suggests that the malignant phenotypes of cancers are determined not only by the intrinsic properties of cancer cells but also by components in the tumor microenvironment (TME). In this study, we comprehensively characterized the TME of cutaneous melanoma (CM). As a result, tumor stage, tissue site, ulceration, thickness as well as patient age, sex were associated with immune infiltration. Patients of higher immune infiltration exhibited better survival outcomes, and antitumor effector cells, such as CD8 T cells and M1 macrophages, were found in significantly higher numbers in those tissues. Differential expression of mRNAs and long non-coding RNAs (IncRNAs) was analyzed and utilized to construct an immune-related competing endogenous RNA network, in which a IncRNA-associated subnetwork that could positively regulate the expression of IFN- $\gamma$ was highlighted. Functional analysis confirmed that this network was remarkably enriched in functional terms related to both immune response and tumor-intrinsic pathways. Finally, a total of 109 high-confidence prognostic genes were identified, and a gene module that contained several key immune checkpoint molecules or modulators (PD-1, PD-L1, PD-L2, and LCK) was screened, which confers survival benefit for CM patients as supported by both overall and relapse-free survival rates from different datasets.
\end{abstract}

Key words: cutaneous melanoma, tumor microenvironment, immune infiltration, competing endogenous RNA, prognosis

\section{Introduction}

Cutaneous melanoma $(\mathrm{CM})$ is the most common type of malignancy arising from melanocytes. It is also the most aggressive skin cancer that causes approximately 60,700 deaths worldwide annually, accounting for more than $80 \%$ of skin cancer-related deaths [1]. For most limited-stage melanomas, surgical resection of the primary tumor is a standard and successful treatment, while for extensive-stage cases, treatment modalities are more complicated because most single or even combination therapies are effective in only a subset of patients [2]. Despite the encouraging clinical results of novel therapies, the prognosis of advanced cases remains unsatisfactory with the 5-year survival rate slightly above $20 \%$ [3].

Over the past decade, there has been increasing interest in understanding the roles of the immune system in the initiation and progression of cancer. Tumor microenvironment (TME) has become the focus of attention because it consists of both cancer cells and nonmalignant stromal cells, including various types of immune cells. The activation state and components of tumor-infiltrating immune cells are important parameters that influence tumor biology and predict tumor prognosis. For example, cytotoxic CD8 $\mathrm{T}$ cells and CD4 helper T cells target antigenic tumor cells to prevent tumor growth [4]. A 
high level of activated CD8 T cells is associated with prolonged patient survival time in many cancers, including CM [5]. Conversely, tumor-associated macrophages, mast cells and neutrophil granulocytes exert effects on promoting tumor progression, and the intense infiltration of these cells generally indicates poor prognosis [5].

In addition, tumor-infiltrating immune cells serve as potential drug targets. Recently, a new therapeutic strategy, known as immune checkpoint blockade, has been developed, aiming at restraining the molecular interplay between tumor cells and immune cells. Immunotherapy with several checkpoint blockers targeting programmed cell death protein 1 (PD-1), PD-1 ligand (PD-L1), or cytotoxic T lymphocyte antigen-4 (CTLA-4) has resulted in significant improvements in the clinical outcomes of patients with various types of cancers including melanoma [6]. However, not all CM patients benefit from these drugs. A subset of patients who initially respond to immunotherapy later develop disease progression, suggesting the existence of intrinsic immune resistance [2]. In previous studies, PD-L1 expression, tumor mutation burden, neoantigen load and deficient DNA mismatch repair have been associated with the immunotherapeutic responsiveness of cancer [7-10]. However, none of these features alone have been validated as sufficient predictors [11]. More importantly, TME and infiltrating immune cells are unique to each cancer type; thus, studying changes of immune infiltration with the associated molecular expression and interactive features based on an individual basis is crucial in elucidating the mechanisms underlying immune evasion by melanoma and immune checkpoint blockades.

In this study, based on a large-scale computational analysis of expression profiles of 879 $\mathrm{CM}$ tissues from The Cancer Genome Atlas (TCGA), Gene Expression Omnibus (GEO) and ArrayExpress, we comprehensively characterized the tumor immune microenvironment of $\mathrm{CM}$ from three different levels: the tissue level, cellular level and molecular level. The relationship between immune infiltration and clinicopathological characteristics as well as immune cell composition was explored. Both protein-coding genes and long noncoding RNAs (lncRNAs) that were associated with immune functions were identified and incorporated for network analysis. By performing Kaplan-Meier survival analysis with the TCGA cohort and in an independent dataset, we eventually discovered an immune-related gene module that conferred prognostic benefit for $\mathrm{CM}$ patients. The results are expected to generate novel insights into the tumor immune microenvironment of $\mathrm{CM}$ and provide potential biomarkers for clinical use.

\section{Materials and Methods}

\section{Data collection}

The level 3 sequencing data of mRNA, lncRNA and miRNA of the CM tissues were obtained from the TCGA database via the TCGAbiolinks package [12]. For long RNAs, both raw counts and fragments per kilobase million (FPKM) data were collected. The datasets contained 468 samples, one for each patient. For miRNAs, only raw count data were obtained, and the dataset contained 448 samples. The associated clinical information of the patients and genomic subtypes was retrieved from the UCSC Xena database [13]. For validation, the processed expression meta-dataset of $194 \mathrm{CM}$ samples was downloaded from ArrayExpress with E-MTAB-6697. Another dataset that contained $214 \mathrm{CM}$ samples was downloaded from GEO with GSE65904 [14], in which the outcome and relapse-free survival (RFS) time of the patients were incorporated. A $\log 2$ transformation was applied to the processed expression data before further analysis.

\section{ESTIMATE}

The infiltration of noncancerous cells in tumor tissues was assessed by ESTIMATE based on expression profile of 141 immune-related genes [15]. The stromal, immune and ESTIMATE scores of the tumor tissues from 25 cancer types used in this study were computed using the RNA-seq V2 data from TCGA [15]. The immune scores of the CM samples from the E-MTAB-6697 and GSE65904 datasets were calculated from the normalized expression data using the R package ESTIMATE [15].

\section{CIBERSORT estimation}

Normalized gene expression data were used to infer the relative proportions of 22 immune cell types using CIBERSORT [16]. The FPKM data of 466 tumor samples (2 samples with no available immune scores were excluded from this analysis) from the TCGA, or the processed expression data from E-MTAB-6697 and GSE65904 were used as input and the LM22, which contained 547 genes that accurately differentiate 22 individual human hematopoietic cell types, was used as a reference gene signature. Permutations were set to 1,000 . Only samples with a CIBERSORT $P<0.05$ were considered eligible for further analysis.

\section{Differential gene expression analysis}

The differentially expressed mRNAs (DEmRNAs) and lncRNAs (DElncRNAs) were identified using edgeR [17], with | $\log 2$ fold changes | $>1$ and FDR < 0.05 considered significant. Molecules 
with average read counts greater than 1 were included for this analysis.

\section{Construction of the competing endogenous RNA (ceRNA) network}

The ceRNA network was constructed using the "gdcCEAnalysis" function of the GDCRNATools package [18]: (1) the lncRNA and mRNA must share a significant number of miRNAs (hypergeometric test, $\mathrm{P}<0.05)$; (2) the expression of the lncRNA and mRNA must be positively correlated (Pearson correlation, $\mathrm{P}<0.05)$; and (3) those common miRNAs should play similar roles in regulating the expression of the lncRNA and mRNA (regulation similarity $>0$ ). The raw count data of both miRNA and long RNA molecules were normalized by the "gdcVoomNormalization" function. All DEmRNAs and DElncRNAs were kept for this analysis, together with 523 miRNAs whose average read counts were greater than 10. The interactions between miRNAs and mRNAs that are supported by strong experimental evidence were obtained from miRTarBase [19], we obtained 8,377 unique miRNA-mRNA interactions. For miRNA-lncRNA regulations, a total of 73,087 nonredundant miRNA-lncRNA interactions supported by experimental evidence were downloaded from LncBase [20].

\section{Enrichment analysis}

The first 300 upregulated or downregulated genes identified from the differential expression analysis were used in the functional enrichment analysis with the plug-in software for Cytoscape [21], ClueGO v_2.5.4 [22]. The terms from Gene Ontology (GO), Kyoto Encyclopedia of Genes and Genomes (KEGG), WikiPathways and Reactome were analyzed. The GO term fusion option was selected. The significance value was set as $\mathrm{P}$-value $<0.05$, and the Bonferroni (two-sided hypergeometric test) was used as the multiple test correction. The threshold of the $\mathrm{K}$ score that reflects the association between two terms was set at 0.4 , and similar terms were given the same color. GO analysis of the ceRNA network and protein-protein interaction (PPI) module was performed using the web-based Enrichr tool [23], with the criterion of adjusted P-value $<0.05$.

\section{Statistical analysis}

In this study, unpaired two-sided t-test or the Wilcoxon signed rank test was used to make statistical comparisons between two patient groups. For a comparison of more than two groups, one-way ANOVA was utilized. All tests were carried out using Prism 8.0 (GraphPad, San Diego, USA) or R (version 3.6.0, Auckland, NZ). A P-value less than 0.05 was considered statistically significant for all statistical analyses if not otherwise specified.

\section{Survival analysis}

Kaplan-Meier survival analysis was performed using the R package "survival" [24]. Patients with survival times less than 30 days were filtered out beforehand, and the remaining patients were classified into high and low groups based on the median statistics. Survival curves were plotted using the "survfit" function in the survival package, and the differences in survival curves for the two groups were analyzed with the log-rank method using the function "survdiff".

\section{PPI network analysis}

The PPI network was constructed using the web-based Search Tool for the Retrieval of Interacting Genes/Proteins (STRING) database [25]. The PPI pairs with an interaction score $>0.4$ were considered as significant and used for construction of the network. The MCODE plug-in from Cytoscape was then used to extract modules from the PPI network with default parameters [26].

\section{Results}

\section{Tissue-level immune infiltration in $\mathrm{CM}$ and its clinical relevance}

In this study, the microenvironment of tumor tissues was assessed by the ESTIMATE algorithm based on two major types of the nontumor components, immune cells and stromal cells [15]. Compared with other tumor types, the infiltration of noncancerous cells in CM was relatively prominent, particularly the immune cells, with average enrichment scores that ranked the 6th of all 25 evaluated cancers (Fig. S1). We next explored the correlation between the scores and clinicopathological characteristics. Briefly, younger patients generally had higher immune and stromal scores than older patients (Fig. 1A; $\mathrm{P}=0.0477$ vs. $\mathrm{P}=0.0034$ ). Female patients had higher immune and stromal scores than male patients, although the latter case was not statistically significant (Fig. $1 \mathrm{~B} ; \quad \mathrm{P}=0.0372$ vs. $\mathrm{P}=0.5827$ ). Extensive-stage cases exhibited significantly higher immune and stromal scores than early stage cases (Fig. $1 C$; $P=0.0275$ vs. $P=0.0072$ ), as did the tumors of metastatic sites compared with those of primary sites (Fig. 1D; $\mathrm{P}<0.0001$ ). Tissues with ulceration or higher Clark levels had both lower immune and stromal scores (Figs. 1E-1F). As for genomic subtypes [27], the BRAF mutant cases had the highest stromal scores, followed by triple-wild type, NF1 mutant and RAS mutant cases (Fig. 1G; $\mathrm{P}=0.019)$. The immune scores of the BRAF subtype 
also ranked the highest of all four subtypes, although not statistically significant $(\mathrm{P}=0.2497)$. The association of immune infiltration with patient survival was analyzed. Kaplan-Meier survival curves showed that the cases with higher immune infiltration (immune_H) conferred prognostic benefit in both overall survival (OS) and RFS compared to those with lower immune infiltration (immune_L) (Figs. 1H-1I; $\mathrm{P}<0.0001$ ), while no survival difference was observed between the samples with variable stromal infiltration (stromal_H and stromal_L). Collectively, these findings suggested that the TME, particularly immune cell infiltration, could have important effects on the pathogenesis of $\mathrm{CM}$ and clinical outcomes of patients.

\section{Characterization of the immune cell composition in CM}

The CIBERSORT method was applied to characterize the cellular composition of the tumor-infiltrating immune cells in CM tissues [16]. A total of 305 samples from the TCGA dataset were successfully deconvolved. Figure 2A shows that, on average, the M0 macrophages (19.22\%) were the most abundant immune infiltrates, followed by CD8 T cells (18.29\%), plasma cells (12.58\%), M2 macrophages

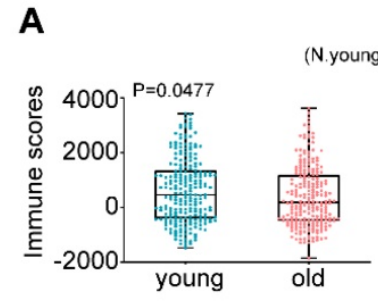

Age

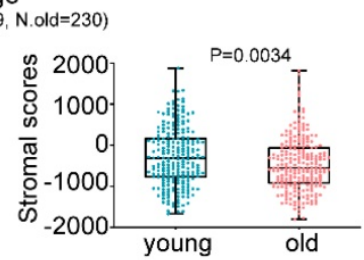

B

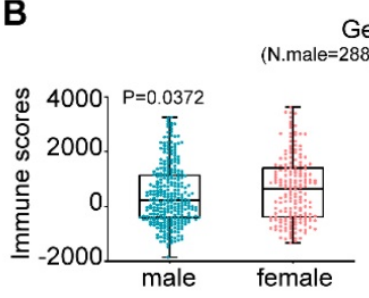

Gender

female $=179$

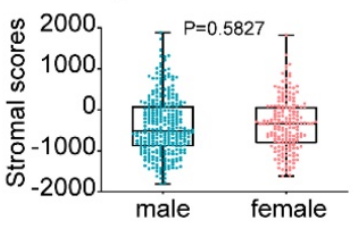

D

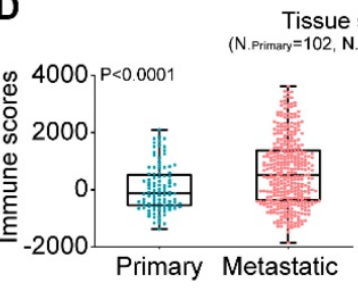

Tissue sites

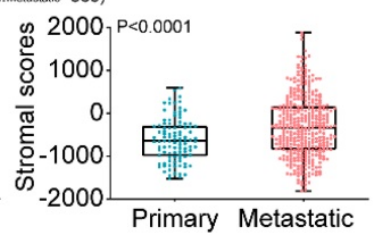

F

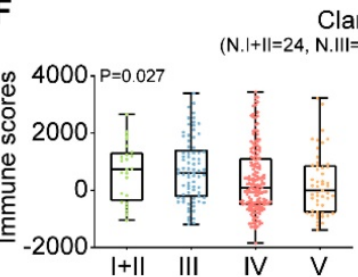

Clark's leve

$$
77, N . I V=167, N . V=51 \text { ) }
$$

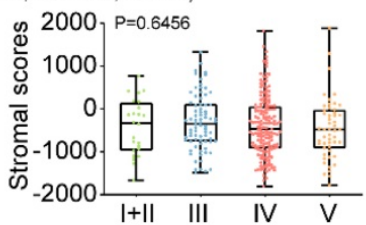

H
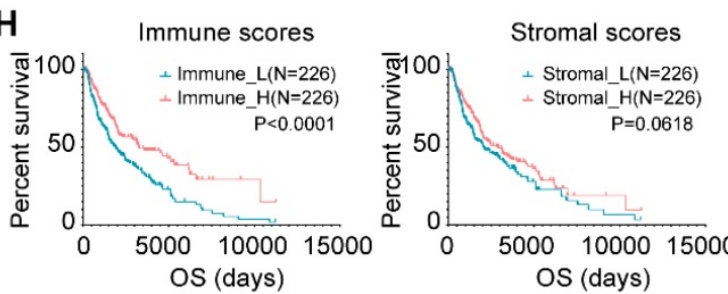

C

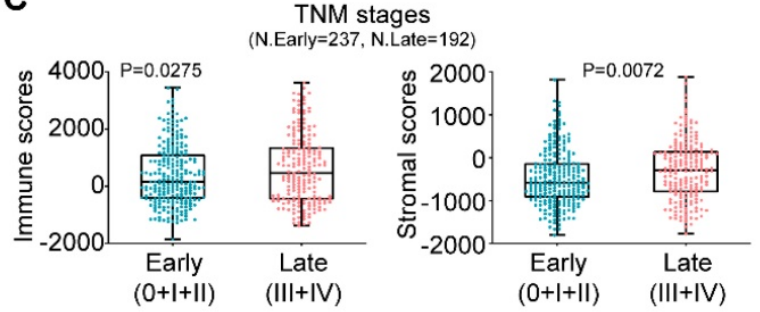

E
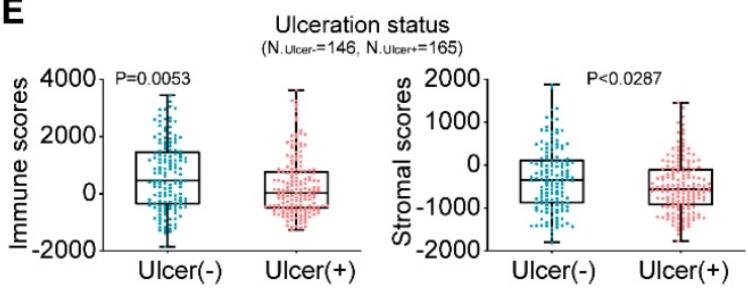

G
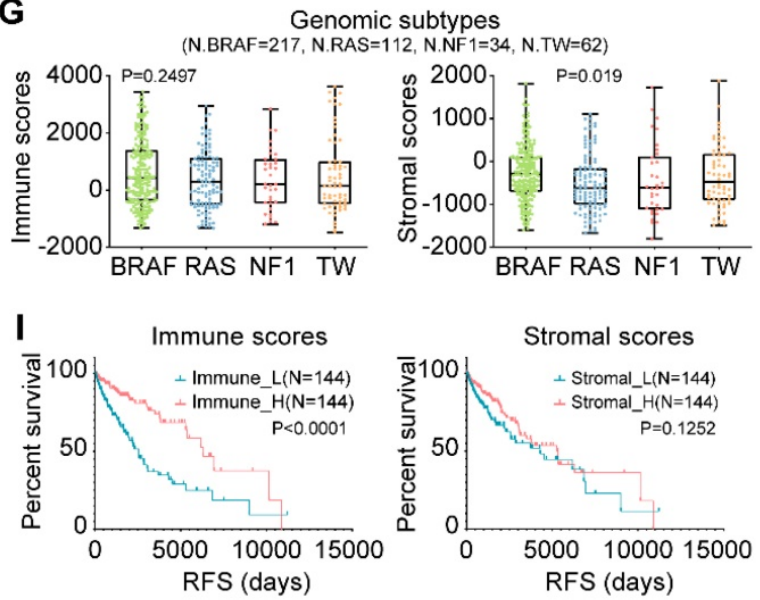

Figure 1. Associations between TME and clinicopathological features in CM. (A-G) Correlation of the immune and stromal scores with age, sex, TNM stage, tissue sites, ulceration status, Clark's level stage and genomic subtypes. $(\mathrm{H}-\mathrm{l})$ Correlation of the immune and stromal scores of CM tissues with the OS or RFS of the patients as illustrated by Kaplan-Meier survival curves. Patients were divided into two groups based on their median scores. P-values and the number of cases in each subgroup of the above analyses (A-I) are displayed. OS, overall survival; RFS, relapse-free survival; TW, triple-wild type. 
(11.95\%) and M1 macrophages (6.58\%). The same analysis was performed on two additional datasets, namely, GSE65904 and E-MTAB-6697, we found that the fractions of M0/M1/M2 macrophages and CD8 T cells were relatively stable across those datasets while some other cell fractions exhibited more variable, such as plasma cells, activated mast cells and neutrophils (Fig. S2), suggesting the heterogeneity of the immune infiltration of the TME. Correlation analysis based on the TCGA data suggested that the number of M0 macrophage cells was inversely related to that of CD8 $\mathrm{T}$ cells $\left(\mathrm{r}^{2}=-0.56\right)$, plasma cells $\left(\mathrm{r}^{2}=-0.38\right)$, follicular helper $\mathrm{T}$ cells $\left(\mathrm{r}^{2}=-0.35\right), \mathrm{CD} 4$ memory activated $\mathrm{T}$ cells $\left(r^{2}=-0.34\right)$ and M1 macrophage cells $\left(r^{2}=-0.29\right)$, indicating that functional antagonism might exist between M0 macrophages and those cells.
A

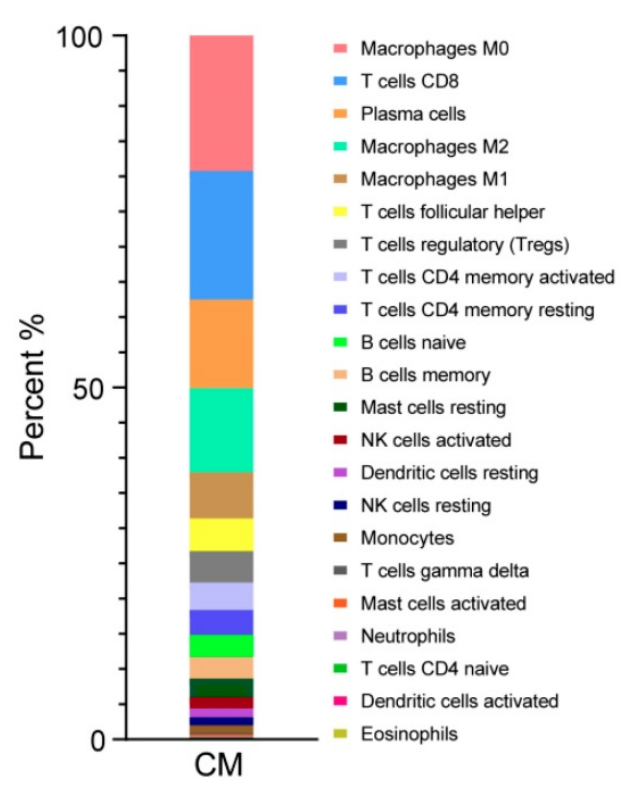

B

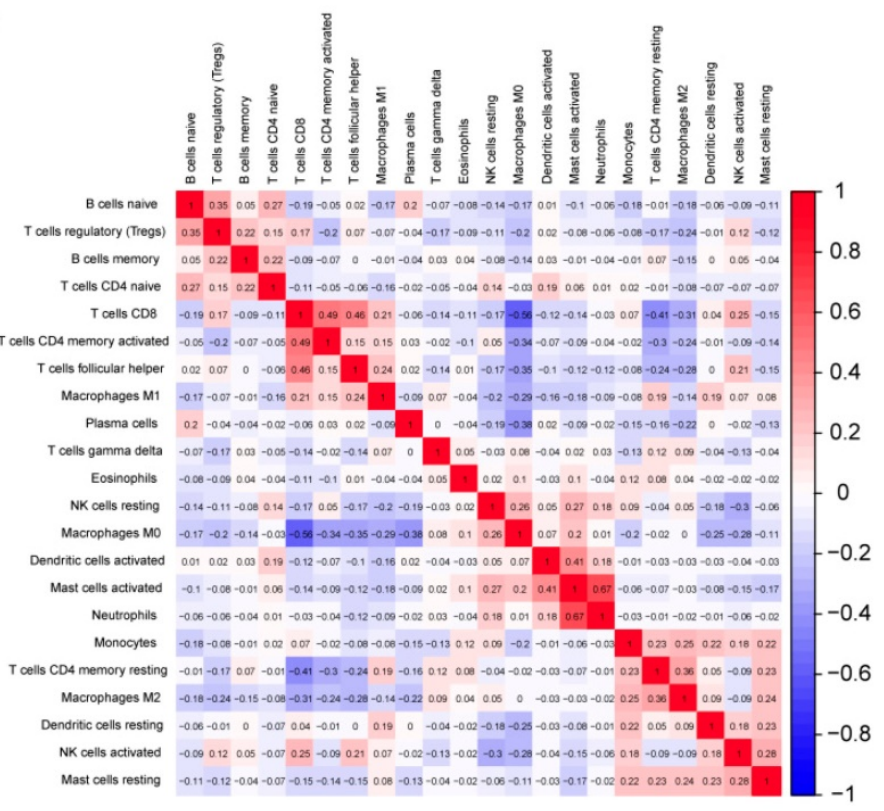

C

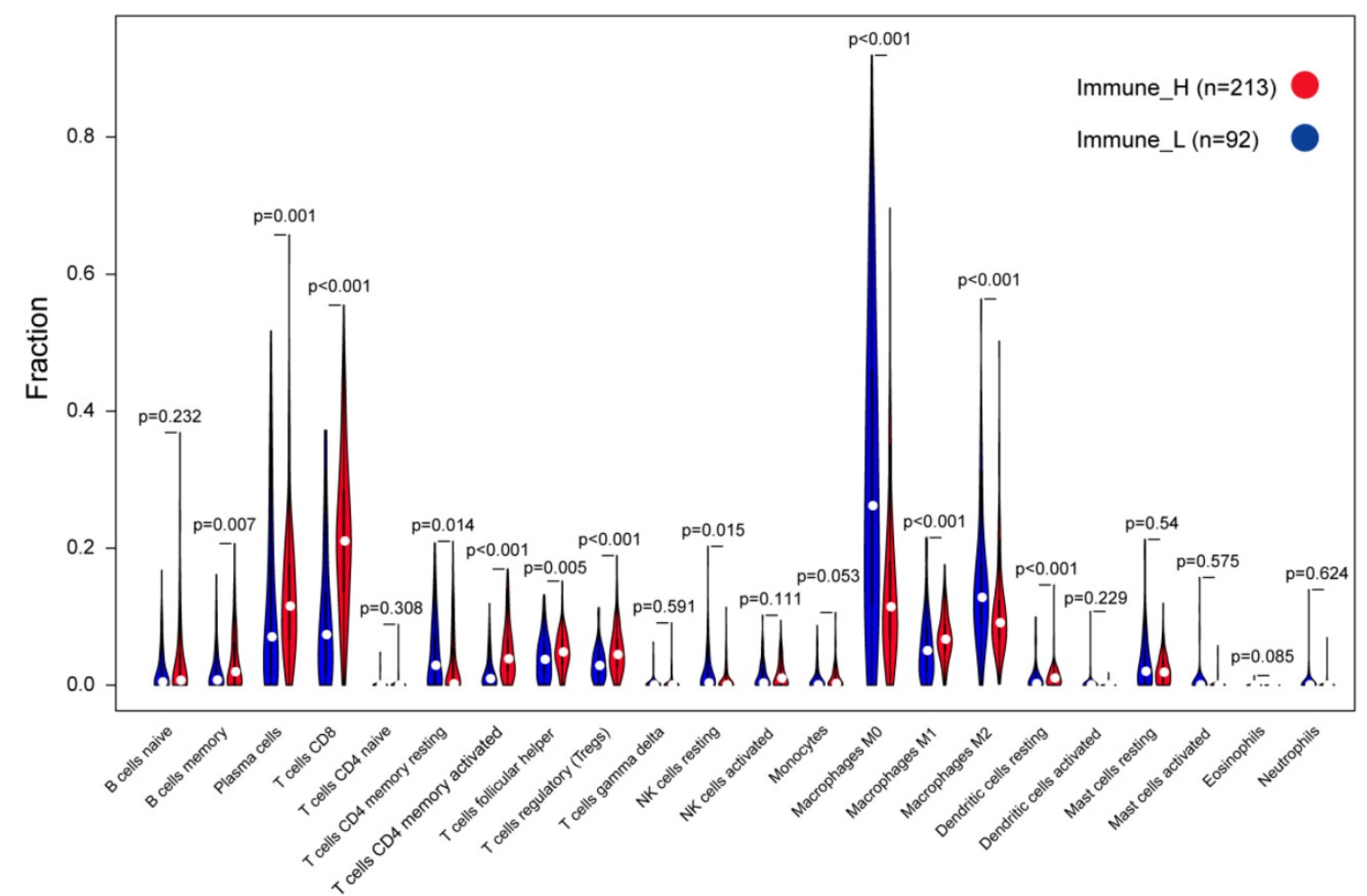

Figure 2. Distribution of immune cell fractions in CM tissues based on TCGA data. (A) The percentage of 22 types of immune cell subsets. (B) Correlation of 22 types of immune cell subsets. (C) Comparison of the immune cell fractions between tumor tissues of the immune_H and immune_L groups. 
In contrast, a highly positive correlation between activated mast cells and neutrophils or activated dendritic cells $\left(r^{2}=0.67\right.$ or 0.41$)$ suggested that these cells might function synergistically in tumor stroma (Fig. 2B). Comparison of the immune cell subsets between different immune infiltration levels revealed that CD8 T cells, activated memory CD4 T cells, plasma cells and M1 macrophages were present in significantly higher numbers in immune_H than in immune_L ( $\mathrm{P} \leq 0.001$, Wilcoxon signed rank test), while M0 and M2 macrophages were more abundant in the immune_L group ( $\mathrm{P}<0.001$; Fig. $2 \mathrm{C})$. These results were largely confirmed in the ArrayExpress and GEO datasets (Figs. S3-S4). Kaplan-Meier analysis showed that naïve B cells were associated with shorter OS time, while M1 macrophages were associated with an improved outcome; Based on RFS, CD8 T cells, activated memory CD4 T cells and follicular helper T cells were correlated with longer survival time, whereas resting memory $\mathrm{CD} 4 \mathrm{~T}$ cells indicated the opposite $(\mathrm{P}<0.05 ;$ Fig. S5). In summary, despite the variance of the immune cell composition from different studies, the antitumor immune activity of the immune_H group was enhanced.

\section{Association between molecular expression and immune infiltration}

To identify the molecular traits that were associated with immune infiltration, we performed differential expression analysis by comparing tumor samples of the immune_H and immune_L groups from the TCGA cohort. As shown by the volcano maps (Fig. 3A), 1,553 mRNAs and 530 lncRNAs were found to be upregulated in the tissues from the immune_H group, while 801 mRNAs and 279 IncRNAs were downregulated. The top 100 dysregulated RNAs are provided in Table S1-S2. To gain mechanistic insights into the dysregulation between the two groups, we performed a functional analysis of the top upregulated or downregulated genes using ClueGO [22]. As a result, 104 functional terms were significantly enriched for the upregulated genes (Table S3). These terms were clustered into 14 groups, the majority of which were closely related to the activation of immune responses (Fig. 3B), such as human immune response (adjusted $\mathrm{P}=3.78 \mathrm{E}-70$ ), regulation of $\mathrm{T}$ cell activation (adjusted $\mathrm{P}=2.62 \mathrm{E}-5$ ), immunoregulatory interactions between a lymphoid and a non-lymphoid cell (adjusted $\mathrm{P}=1.18 \mathrm{E}-07$ ) and lymphocyte activation (adjusted $\mathrm{P}=2.86 \mathrm{E}-43$ ). In contrast, terms enriched in the downregulated genes were mainly related to skin development and structures (Fig. S6 \& Table S4). The results were consistent with the observation that the immune cells were largely infiltrated in those immune_H tumor samples.

\section{Construction of an immune infiltration-related ceRNA network in CM}

To investigate the role of lncRNAs and their interactions with protein-coding genes in the infiltration of immune cells in CM, we constructed a ceRNA network based on the DEmRNAs and DElncRNAs identified above using GDCRNATools [18]. The network consisted of 47 lncRNAs, 86 miRNAs and 138 mRNAs with a total of 375 interactions (Table S5). GO analysis confirmed that both immune responses and tumor-intrinsic pathways were significantly enriched in the network (Fig. 4A), such as cellular response to cytokine stimulus, positive regulation of MAPK cascade and protein tyrosine kinase activity. With Cytoscape, the network was arranged into three layers, in which the lncRNAs of the inner layer could modulate the expression of the mRNAs of the outer layer through interactions with the miRNAs of the intermediate layer (Fig. 4B). Notably, 95.3\% (82/86) of the miRNAs were denoted to be associated with melanoma by Mammal NcRNA-Disease Repository (MNDR) v2.0 (confidence score $>0.4$ ) [28]. An examination of the degree distribution of these RNAs revealed a power-law with a slope of -0.747 and $R^{2}=0.932$, suggesting a typical scale-free structure of biological network. Indeed, the top 10 lncRNAs with highest degrees (hub lncRNAs) controlled more than 78\% $(108 / 138)$ of the genes in this network through competitive binding of 63 miRNAs (Table 1).

Table 1.

\begin{tabular}{|c|c|}
\hline ncRNAs & miRNAs \\
\hline RP11-588K22.2 & $\begin{array}{l}\text { hsa-miR-361-5p, hsa-miR-195-5p, hsa-let-7e-5p, hsa-miR-21-5p, } \\
\text { hsa-let-7i-5p, hsa-miR-19a-3p, hsa-miR-19b-3p, hsa-miR-9-5p, } \\
\text { hsa-miR-34a-5p, hsa-let-7a-5p, hsa-let-7b-5p, hsa-let-7f-5p, } \\
\text { hsa-miR-15a-5p, hsa-miR-126-5p, hsa-miR-454-3p, hsa-miR-16-5p, } \\
\text { hsa-miR-182-5p, hsa-miR-98-5p, hsa-miR-135a-5p, hsa-let-7c-5p, } \\
\text { hsa-miR-301b-3p }\end{array}$ \\
\hline RP11-284N8.3 & $\begin{array}{l}\text { hsa-miR-24-3p, hsa-miR-361-5p, hsa-miR-126-5p, hsa-miR-19a-3p, } \\
\text { hsa-miR-19b-3p, hsa-miR-27a-3p, hsa-miR-23b-3p, hsa-miR-101-3p, } \\
\text { hsa-miR-23a-3p, hsa-miR-26a-5p, hsa-miR-27b-3p, hsa-miR-485-3p, } \\
\text { hsa-miR-338-3p, hsa-miR-9-3p }\end{array}$ \\
\hline RP6-24A23.7 & $\begin{array}{l}\text { hsa-miR-24-3p, hsa-miR-34a-5p, hsa-miR-34b-5p, hsa-miR-34c-5p, } \\
\text { hsa-miR-449a, hsa-miR-629-5p, hsa-miR-15a-5p, hsa-miR-16-5p, } \\
\text { hsa-miR-491-5p, hsa-miR-144-3p, hsa-miR-29a-3p, hsa-miR-29b-3p, } \\
\text { hsa-miR-29c-3p }\end{array}$ \\
\hline RP1-60O19.1 & $\begin{array}{l}\text { hsa-miR-195-5p, hsa-miR-199a-5p, hsa-miR-150-5p, } \\
\text { hsa-miR-218-5p, hsa-miR-15a-5p, hsa-miR-15b-5p, hsa-miR-16-5p, } \\
\text { hsa-miR-29b-1-5p, hsa-miR-145-5p, hsa-miR-214-3p, } \\
\text { hsa-miR-338-3p }\end{array}$ \\
\hline RP11-79H23.3 & $\begin{array}{l}\text { hsa-miR-361-5p, hsa-miR-181a-5p, hsa-miR-140-3p, } \\
\text { hsa-miR-27a-3p, hsa-miR-23b-3p, hsa-miR-181b-5p, } \\
\text { hsa-miR-181c-5p }\end{array}$ \\
\hline HCP5 & $\begin{array}{l}\text { hsa-miR-92a-3p, hsa-miR-205-5p, hsa-miR-141-3p, hsa-miR-145-5p, } \\
\text { hsa-miR-101-3p, hsa-miR-125a-3p }\end{array}$ \\
\hline CTD-2369P2.8 & $\begin{array}{l}\text { hsa-miR-92a-3p, hsa-miR-25-3p, hsa-miR-32-5p, hsa-miR-363-3p, } \\
\text { hsa-miR-194-5p, hsa-miR-107 }\end{array}$ \\
\hline RP11-54O7.1 & $\begin{array}{l}\text { hsa-miR-16-5p, hsa-miR-24-3p, hsa-miR-9-5p, hsa-miR-107, } \\
\text { hsa-miR-424-5p }\end{array}$ \\
\hline AP001055.6 & hsa-let-7a-5p, hsa-let-7e-5p, hsa-let-7i-5p, hsa-let-7b-5p \\
\hline RP11-203J24.9 & hsa-let-7g-3p, hsa-miR-19a-3p, hsa-miR-19b-3p, hsa-miR-188-5p \\
\hline
\end{tabular}


Of all the genes regulated by hub IncRNAs, IFNG, whose translational output plays a key role in antitumor immunity [29], was among the most upregulated molecules $(\log F C=3.74)$ in the immune_H group while its transcript was under the control of the most miRNA mediators, suggesting that the accumulation of IFNG associated with immune infiltration might be positively modulated by those highly competitive lncRNAs, such as RP11-588K22.2, RP11-284N8.3, RP1-60O19.1 and RP11-79H23.3 (Fig. 5). Importantly, most of the lncRNAs remain uncharacterized in $\mathrm{CM}$, which necessitates further focused studies.

A
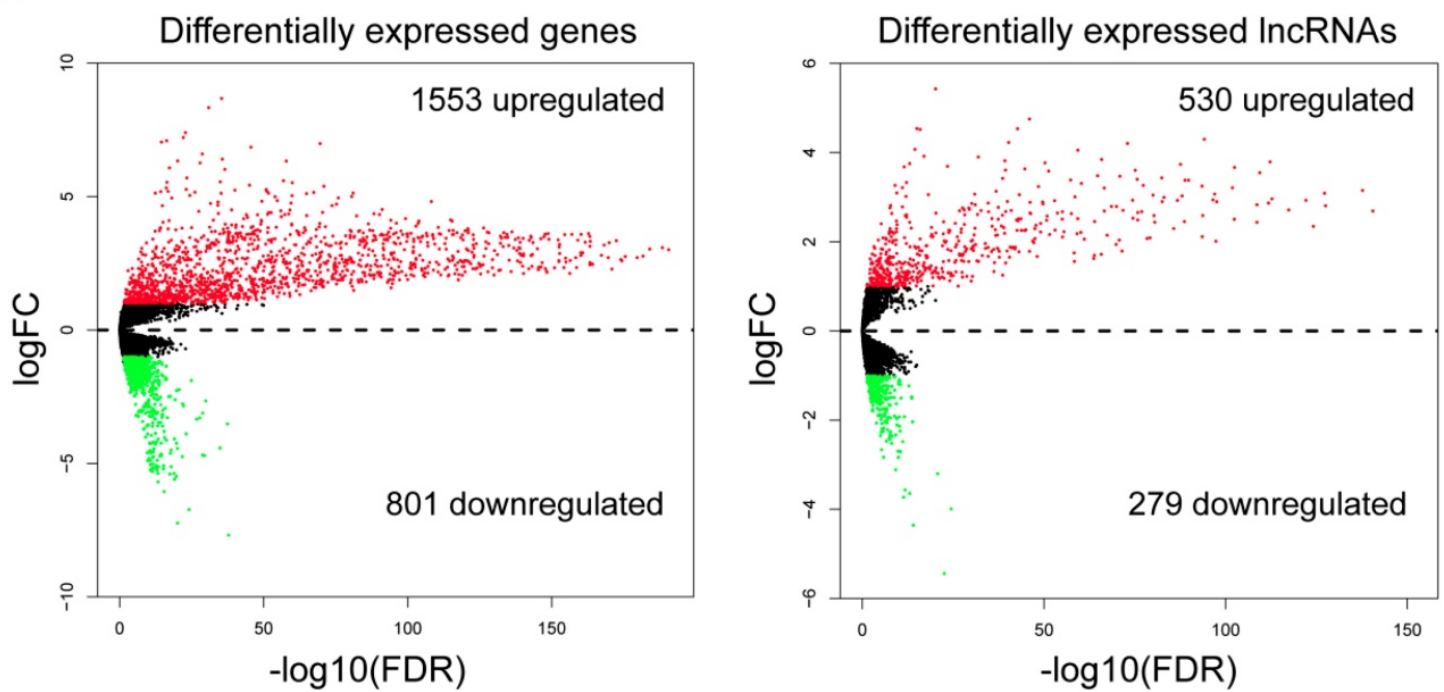

B

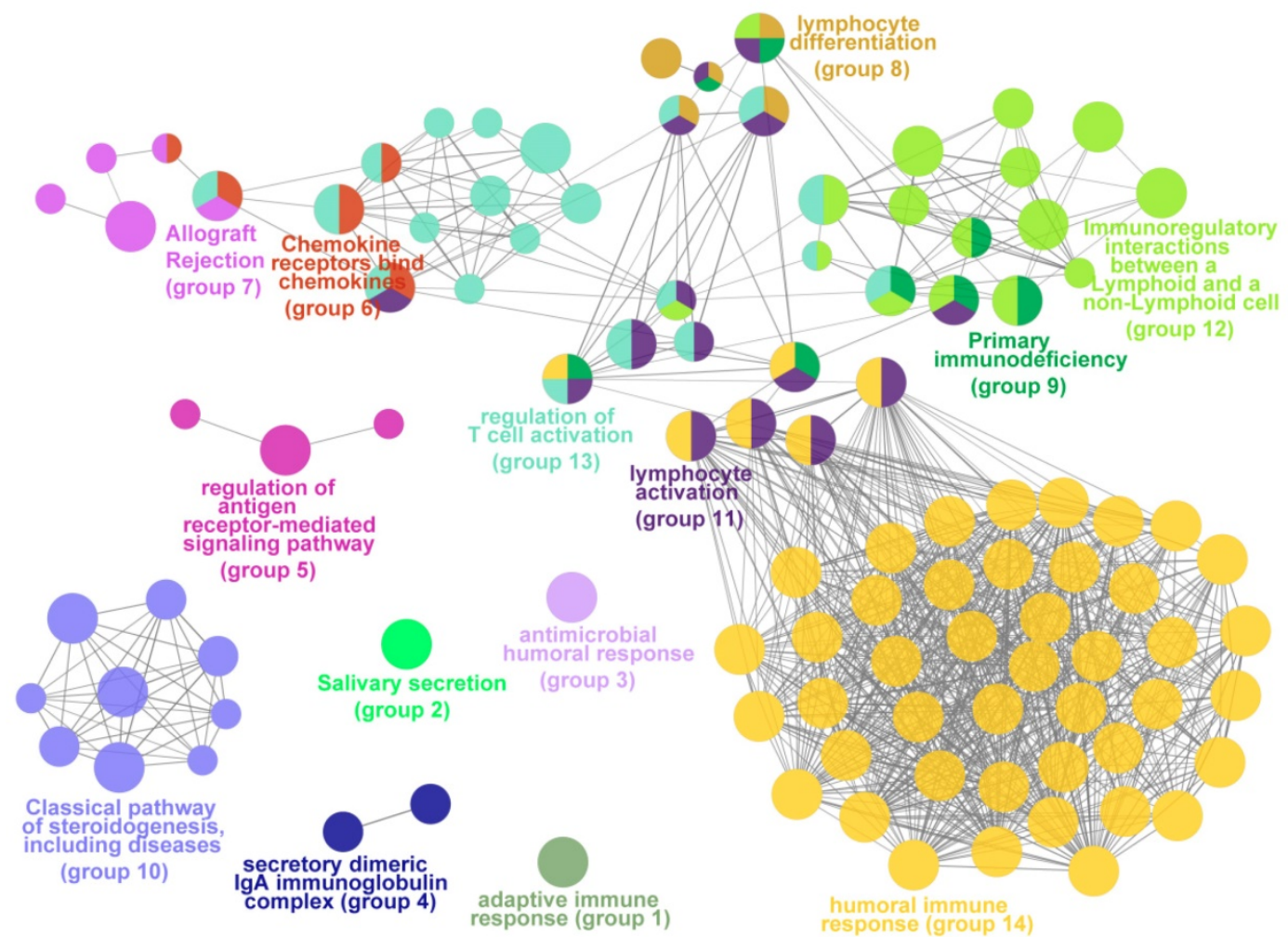

Figure 3. Differential expression analysis and functional annotation. (A) Volcano maps of the differentially expressed mRNAs and IncRNAs. Red points represent the RNAs with a $\log F C>1$ and FDR $<0.05$. Green points represent RNAs with a $\log F C<-1$ and FDR $<0.05$. (B) Grouped network of the functional terms enriched in the upregulated genes in the immune_H tumor samples. The nodes represent the enriched terms (adjusted P-value $<0.05$ ). The size of the nodes reversely represents the statistical significance of the terms. Functionally related groups partially overlap. 
A

Biological Process (BP)

cellular response to cytokine stimulus cytokine-mediated signaling pathway inflammatory response

-regulation of ERK1 and ERK2 cascade -positive regulation of ERK1 and ERK2 cascade positive regulation of MAPK cascade -regulation of cell proliferation

-positive regulation of cytokine production regulation of apoptotic process - positive regulation of protein phosphorylation

$\begin{array}{llllll}0 & 5 & 10 & 15 & 20 & 25\end{array}$ $-\log 10$ (adjusted P)
Molecular Function (MF)

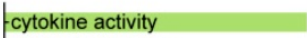

-chemokine activity

chemokine receptor binding

protein tyrosine kinase activity

protein kinase activity

cytokine receptor binding

CCR chemokine receptor binding

C-C chemokine binding

transmembrane receptor protein tyrosine kinase activity -phosphatidylinositol-4,5-bisphosphate 3-kinase activity

$\begin{array}{llll}0 & 5 & 10 & 15\end{array}$ $-\log 10$ (adjusted P)

\section{Cellular Component (CC)}

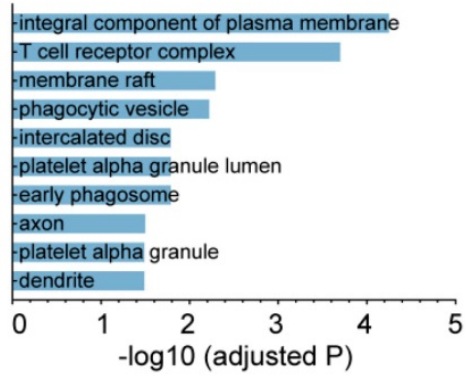

B

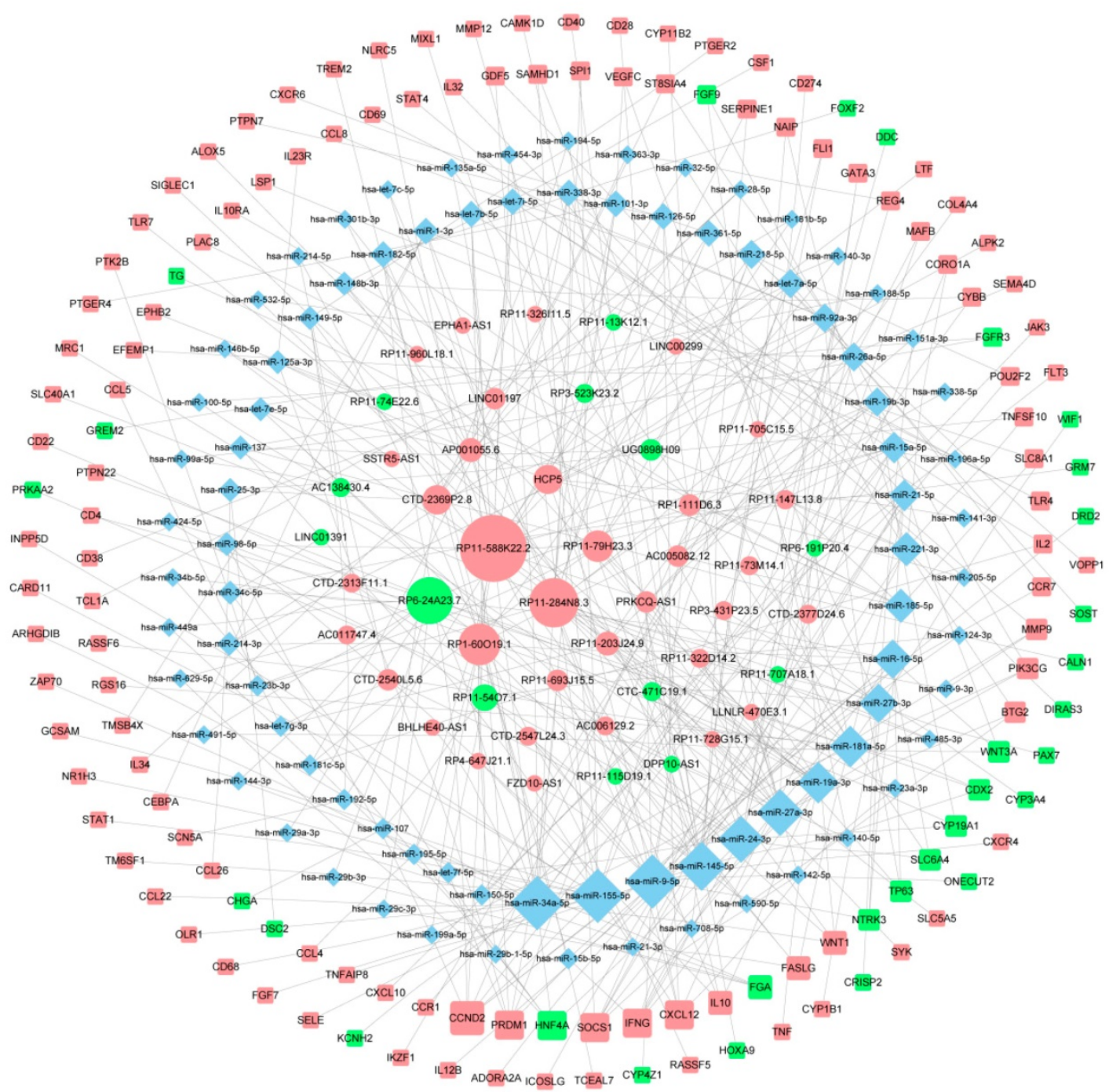

Figure 4. The immune-related ceRNA network. (A) Top $10 \mathrm{GO}$ terms enriched in the ceRNA network. (B) The ceRNA network consisting of 47 IncRNAs, 86 miRNAs and 138 mRNAs was constructed and arranged as a three-layer structure. The ellipses represent IncRNAs, diamonds represent miRNAs and rounded rectangles represent protein-coding genes. The node size is proportional to its degrees. The nodes highlighted in red indicate upregulated expression in the immune_H group, and the nodes labeled green indicate downregulated expression.

\section{Identification of immune-related prognostic module in CM}

To investigate the relationship between immune infiltration and $\mathrm{CM}$ patient prognosis at the molecular level, we performed Kaplan-Meier survival analysis of the 2,354 DEmRNAs from the TCGA dataset. As a result, 715 genes were identified to be associated with both OS and RFS (P<0.01; Fig. 6A), among which 109 genes were further tested and validated in the GSE65904 dataset by Kaplan-Meier estimates of RFS (Table S6). All these molecules appeared to be protective, as patients with higher expression levels of 
these molecules had clearly higher survival rates than those with lower expression levels of these RNAs. To interrogate the interplay among those genes, a PPI network was further constructed (PPI enrichment P-value $<1.0 \mathrm{E}-16)$. The network comprised 84 nodes and 449 edges (Fig. S7). The hub nodes (the top 15\% by degree) included PTPRC, CD86, LCK, LILRB2, CD40LG, TLR8, CD3E, CD274, MNDA, CTSS, HLA-DRA, CXCR3 and FCER1G. These molecules are often central to multiple signaling pathways involved in biological processes like cell proliferation or immune activation, thus influencing more critical functions and making especially attractive drug targets [30]. Indeed, ten of the 13 hub genes have targeted drugs in clinic or clinical trials according to Therapeutic target database (Table S6) [31]. Furthermore, four subnetwork modules were screened from the whole PPI network and the largest module, which consisted of 27 nodes and 146 edges, is shown in Fig. 6B. Several critical immunomodulators such as PD-1, PD-L1 and PD-L2 were involved in this module, and all the module molecules were positively correlated with patient survival, which indicated that this module played a protective role for $\mathrm{CM}$ patients. Figure 6C and 6D illustrates the RFS curves for PD-1, PD-L1 and PD-L2 based on the TCGA and GEO datasets, respectively, with the OS curves displayed in Fig. S8. Functional characterization of this module identified 109 terms from BP, 18 terms from MF and 40 terms from CC that were significantly enriched, the majority of which were closely related to antigen presentation (Fig. 6E). Overall, we discovered a gene module that participates in immune infiltration of $\mathrm{CM}$ tissues and leads to favorable outcomes for the patients.

\section{Discussion}

In this study, we performed a comprehensive assessment of the tumor immune microenvironment of $\mathrm{CM}$ at three different levels. First, we analyzed the association between immune infiltration and clinicopathological characteristics by considering the TME as a whole (tissue level). Next, we explored the cellular composition of the immune infiltrates in individual tumor tissues to establish potential connections between immune cell subsets and clinical outcomes (cellular level). Finally, we focused on the molecules whose expression profiles were correlated with immune infiltration to discover systems-level regulatory mechanisms and potential prognostic biomarkers (molecular level).

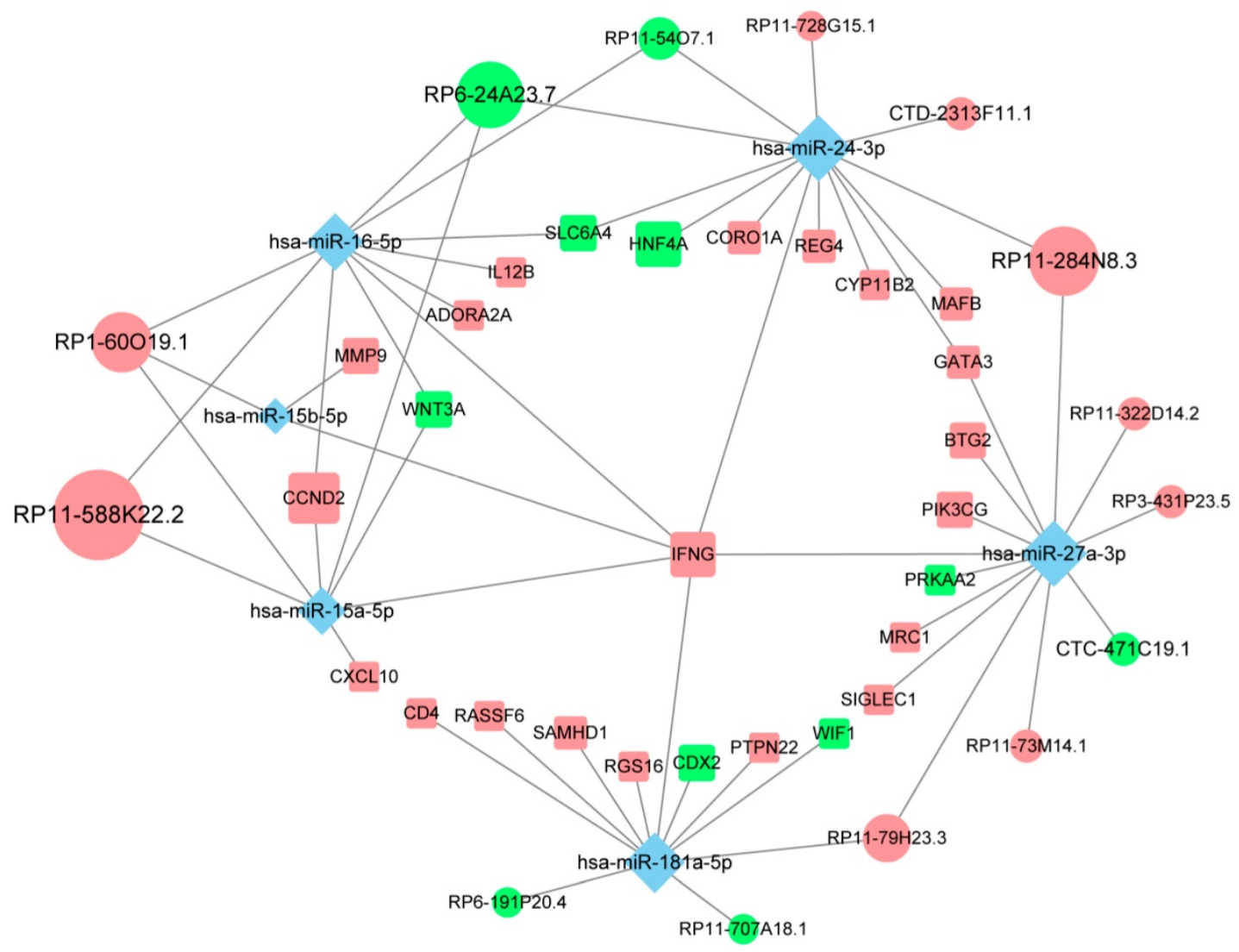

Figure 5. The competing endogenous subnetwork involving IFNG. The ellipses represent IncRNAs, diamonds represent miRNAs and rounded rectangles represent protein-coding genes. The node size is proportional to its degrees across the whole network. The nodes highlighted in red indicate upregulated expression in the immune_H group, and the nodes labeled green indicate downregulated expression. 
A

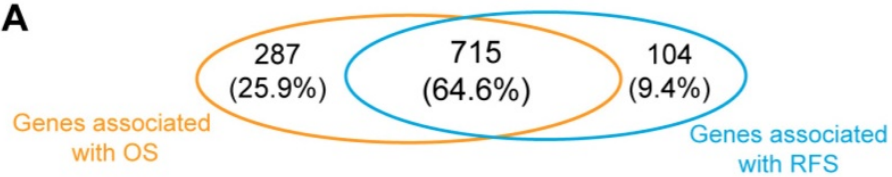

B

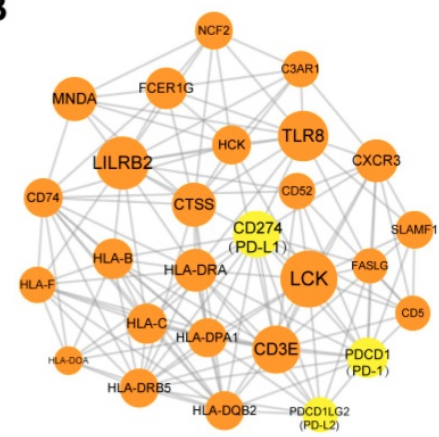

C
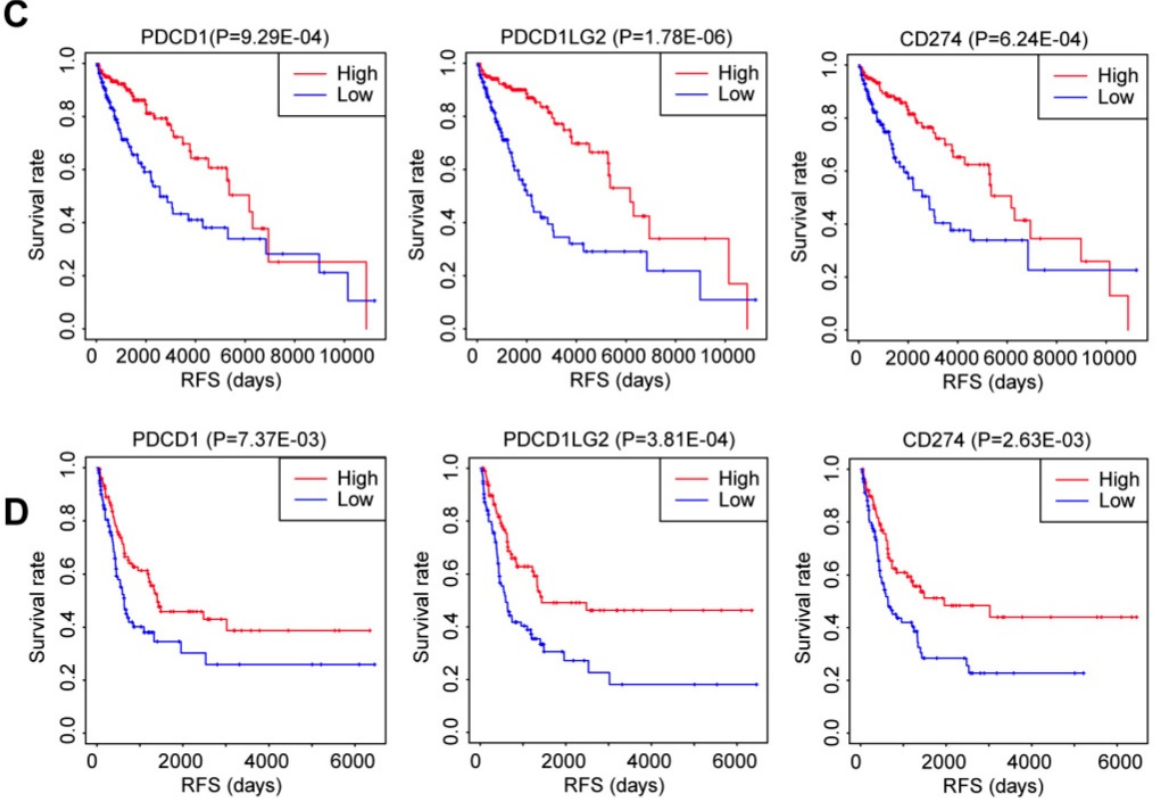

$\mathbf{E}$

Biological Process (BP)

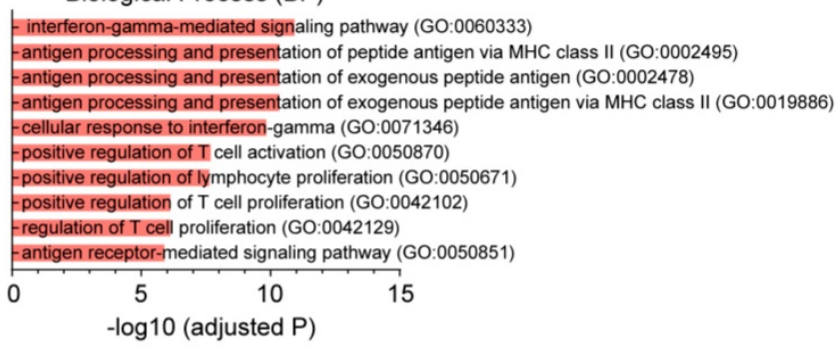

Molecular Function (MF)

-MHC class II receptor activity (GO:0032395)

-MHC class II protein complex binding (GO:0023026)

-MHC protein complex binding (GO:0023023)

- T cell receptor binding (GO:0042608)

-CD4 receptor binding (GO:0042609)

-MHC protein binding (GO:0042287)

-phosphotyrosine residue binding (GO:0001784)

-protein phosphorylated amino acid binding (GO:0045309)

-non-membrane spanning protein tyrosine kinase activity (GO:0004715) - amyloid-beta binding (GO:0001540)

$\begin{array}{llllll}0 & 2 & 4 & 6 & 8\end{array}$
Cellular Component (CC)

-MHC protein complex (GO:0042611)

-integral component of lumenal side of endoplasmic reticulum membrane (GO:0071556)

-ER to Golgi transport vesicle membrane (GO:0012507)

- MHC class II protein complex (GO:0042613)

-COPII-coated ER to Golgi transport vesicle (GO:0030134)

-integral component of endoplasmic reticulum membrane (GO:0030176)

-clathrin-coated endocytic vesicle membrane (GO:0030669)

-lysosomal membrane (GO:0005765)

-Golgi membrane (GO:0000139)

- clathrin-coated endocytic vesicle (GO:0045334)

$\begin{array}{lllll}1 & 5 & 10 & 15 & 20\end{array}$

$-\log 10$ (adjusted P)

Figure 6. Correlation of the immune-related genes with patient prognosis. (A) Venn diagrams showing the number of overlapped prognostic OS and RFS genes ( $<0.01$ ). (B) Selected module from the PPI network constructed using prognostic genes validated by an independent dataset. The key immunomodulators were labeled yellow. (C\&D) Kaplan-Meier RFS curves of the immunomodulators in the (C) TCGA and (D) GEO datasets. (E) Top 10 GO terms enriched in the module. OS, overall survival; RFS, relapse-free survival.

At the tissue level, the correlation between the immune infiltration and clinicopathological characteristics was analyzed. The results suggested that both immune and stromal enrichment status was affected by sex and age in CM. Indeed, the T cell repertoire is generally accepted to decline with 
increasing age, and the cumulative incidence rate for immune response to melanoma vaccination in older patients has been recently shown to be significantly lower than that in younger patients [32]. In addition, males with melanoma generally have worse outcomes than females [33], which can partially be explained by the sex-related differences in the interaction between immune function and tumor biology [34]. Our findings that immune infiltration was significantly associated with multiple pathological features, including tumor staging, tumor site, ulceration and tumor thickness, are largely in line with the findings of previous studies [27, 35-37]. We also revealed that immune infiltration, not stromal infiltration, was positively correlated with patient survival.

At the cellular level, we found that the five most common immune cells in CM tissues accounted for up to $70 \%$ of the immune infiltrates. A comparison study of immune cell fractions showed that tumors with higher immune cell infiltration had significantly higher levels of CD8 T cells, activated memory CD4 T cells and M1 macrophages, while lower levels of M0 and M2 macrophages. In general, CD8 T cells are critical antitumor effector cells, while plasma cells play protective roles in the adaptive immune response. Macrophages exhibit distinct functions based on their activation status. The classically activated M1 macrophages are considered inflammatory (antitumor) while the alternatively activated M2 macrophages are believed to have anti-inflammatory (tumor-promoting) functions. Both types of cells are polarized from uncommitted macrophages (M0) depending on different stimuli. The correlation analysis of these cell types in $\mathrm{CM}$ confirmed their functional associations (Fig. 2B). Importantly, these results could largely be validated in independent datasets. Our findings suggested that, despite the heterogeneity in immune cell content across different studies, the immune_H tumors did exhibit enhanced antitumor immunity, which partially explained why patients from this group had an improved clinical outcome. In addition, Kaplan-Meier analysis of the immune cell subsets provided different findings for OS and RFS, which implicated that those immune cells might play a role in preventing the relapse of the disease (Fig. S5B).

At the molecular level, the DEmRNAs and DElncRNAs between immune_H and immune_L groups were identified and used to construct a ceRNA network. As the functional mediators between mRNAs and lncRNAs, $95.3 \%$ of the miRNAs in this network were denoted to be melanoma-associated, validating the biological relevance of the network. Based on topological structure, the 10 hub lncRNAs were able to control up to $80 \%$ of the mRNAs in this network. Particularly, we found that IFNG, which was significantly overexpressed in melanoma tissues with high immune infiltration, was regulated by several of the hub lncRNAs. The controlled expression of IFNG is critical for effective antitumor responses. Most previous studies document that the production of its translational output, IFN- $\gamma$, is mainly regulated at the transcriptional level by activators or inhibitors [38], while the post-transcriptional regulation is typically inhibitory characterized by miRNA-mediated repression or ARE-mediated decay [39]. Our study showed that IFNG could also be positively modulated by competing lncRNAs at the post-transcriptional level, as suggested by the upregulation of these lncRNAs along with IFNG in the immune_H tumors. Functional analysis suggested that the network is not only involved in the regulation of immune-related pathways, but also regulates tumor-intrinsic pathways in the development of cancer. Recent evidence has shown that oncogenic pathways in tumor cells can be activated to regulate the production of several chemokines and cytokines, which can either decrease the recruitment of immune cells or enhance the recruitment of immunosuppressive cells to tumor sites, contributing to immunoresistance in cancers [40]. Indeed, the MAPK pathway in BRAF ${ }^{\mathrm{V} 600 \mathrm{E}}$ mutant melanoma cells contributes to comprised function of dendritic cell (DC) in the TME of CM, and the inhibition of the pathway can reverse the suppression of DC function [41]. It has also been shown that resistance to BRAFV600E inhibitors (BRAFi) in an autochthonous mouse model of melanoma is associated with restoration of myeloid-derived suppressor cells (MDSC) in the TME, initially reduced by BRAFi treatment, and this process relies upon the reactivation of MAPK pathway [42]. Therefore, targeting these oncogenic pathways is a potential strategy for cancer treatment, particularly in combination with immunotherapies such as check point blockers and chimeric antigen receptor- $\mathrm{T}$ cell therapy. A deeper understanding of the regulatory relationships between immune response and tumor-intrinsic pathways would lead to new therapeutic strategies that might benefit more $\mathrm{CM}$ patients.

Finally, we tested the prognostic relevance of the genes associated with immune infiltration. By Kaplan-Meier analysis and cross validation with GEO dataset, 109 genes were found to be significantly associated with survival of $\mathrm{CM}$ patients. The connections between these molecules were interrogated by PPI network analysis, and a highly compact module that conferred prognostic benefit was obtained, which contained several 
immunotherapeutic targets such as PD-1, PD-L1 and PD-L2. We also noticed that LCK was in this module. This gene belongs to the Src family of protein tyrosine kinases and is an integral component of $\mathrm{T}$ cell receptor signaling. Previous study identified that high protein expression of LCK is strongly correlated with favorable outcomes of CM patients, and tumors from the immune transcriptomic subgroup that correlate with pathological lymphocytic infiltration also express elevated levels of LCK protein [27]. Functional analysis suggested that this module participates in antigen presentation.

This study has some limitations. First, the analysis was based on publicly available datasets, in which it was not possible to obtain all relevant information needed for each patient, particularly, the immune-related comorbidities or medication history that might affect the immune microenvironment of that patient. Such patients should ideally be excluded from our study. Second, the results of this study all came from pure bioinformatics analysis. Although we incorporated independent datasets for validation, the results still need to be confirmed by both in vitro and in vivo experiments. Third, the main purpose of this study was to examine the TME from different levels to screen clinicopathological and molecular features that were associated with immune infiltration in $\mathrm{CM}$ and analyzed their potential interconnections. Although we identified some candidate molecules that might impact this process, we did not conduct in-depth analysis on the functions or mechanisms of specific pathways or molecules, which should warrant further focused studies.

\section{Conclusions}

To conclude, we described the immune landscape in detail, suggesting that TME, particularly immune cell infiltration, could have important effects on the pathogenesis of $\mathrm{CM}$ and clinical outcomes of the patients. Our study has identified the clinicopathological features, immune cell subsets, differentially expressed RNAs that are associated with immune infiltration. We also constructed an immune-related ceRNA network in $\mathrm{CM}$, in which several of the hub lncRNAs were shown to potentially increase the production of IFN- $\gamma$. Finally, we discovered a functional module that contained several validated and potential immunomodulators. Each molecule of the module was illustrated to confer survival advantage for $\mathrm{CM}$ patients as supported by OS and RFS rates from different datasets. We believe that our work will advance the understanding of the immune response in the tumor environment and provide valuable resources to explore key molecules and relevant mechanisms related to tumor immunology and immunotherapy in CM.

\section{Supplementary Material}

Supplementary figures and tables. http://www.jcancer.org/v11p3858s1.pdf

\section{Acknowledgements}

\section{Funding}

This work was supported by the National Natural Science Foundation of China: 81802299; the Chinese Academy of Medical Sciences Innovation Fund for Medical Sciences: 2016-I2M-1-001, 2017-I2M-1-005; the National Key Basic Research Development Plan: 2018YFC1312105; and the Beijing Municipal Science \& Technology Commission: Z181100001918002.

\section{Author Contributions}

WP, ZXY, SN, and HJ conceived the idea; WP, $\mathrm{ZXY}$ and $\mathrm{SN}$ performed the computational experiments and analyzed the data with the help of $\mathrm{ZZH}$; WP, ZXY, SN and ZZH prepared the figures and tables; WP, ZXY, SN, and HJ wrote the manuscript; HJ supervised the project. All authors read and approved the final manuscript.

\section{Competing Interests}

The authors have declared that no competing interest exists.

\section{References}

1. Bray F, Ferlay J, Soerjomataram I, Siegel RL, Torre LA, Jemal A. Global cancer statistics 2018: GLOBOCAN estimates of incidence and mortality worldwide for 36 cancers in 185 countries. CA Cancer J Clin. 2018; 68: 394-424

2. Schadendorf D, van Akkooi ACJ, Berking C, Griewank KG, Gutzmer R, Hauschild A, et al. Melanoma. Lancet. 2018; 392: 971-84.

3. Siegel RL, Miller KD, Jemal A. Cancer statistics, 2020. CA Cancer J Clin. 2020; 70: 7-30.

4. Vesely MD, Kershaw MH, Schreiber RD, Smyth MJ. Natural innate and adaptive immunity to cancer. Annu Rev Immunol. 2011; 29: 235-71.

5. Fridman WH, Pages F, Sautes-Fridman C, Galon J. The immune contexture in human tumours: impact on clinical outcome. Nat Rev Cancer. 2012; 12: 298-306.

6. Robert C, Long GV, Brady B, Dutriaux C, Maio M, Mortier L, et al. Nivolumab in previously untreated melanoma without BRAF mutation. N Engl J Med. 2015; 372: 320-30.

7. Snyder A, Makarov V, Merghoub T, Yuan J, Zaretsky JM, Desrichard A, et al. Genetic basis for clinical response to CTLA-4 blockade in melanoma. N Engl J Med. 2014; 371: 2189-99.

8. Rizvi NA, Hellmann MD, Snyder A, Kvistborg P, Makarov V, Havel JJ, et al. Cancer immunology. Mutational landscape determines sensitivity to PD-1 blockade in non-small cell lung cancer. Science. 2015; 348: 124-8.

9. Hugo W, Zaretsky JM, Sun L, Song C, Moreno BH, Hu-Lieskovan S, et al. Genomic and Transcriptomic Features of Response to Anti-PD-1 Therapy in Metastatic Melanoma. Cell. 2016; 165: 35-44.

10. Le DT, Durham JN, Smith KN, Wang H, Bartlett BR, Aulakh LK, et al. Mismatch repair deficiency predicts response of solid tumors to PD-1 blockade. Science. 2017; 357: 409-13.

11. Teng F, Meng X, Kong L, Yu J. Progress and challenges of predictive biomarkers of anti PD-1/PD-L1 immunotherapy: A systematic review. Cancer Lett. 2018; 414: 166-73.

12. Colaprico A, Silva TC, Olsen C, Garofano L, Cava C, Garolini D, et al. TCGAbiolinks: an R/Bioconductor package for integrative analysis of TCGA data. Nucleic Acids Res. 2016; 44: e71. 
13. Goldman M, Craft B, Hastie M, Repečka K, McDade F, Kamath A, et al. The UCSC Xena platform for public and private cancer genomics data visualization and interpretation. bioRxiv. 2019; Available at: https://doi.org/10.1101/326470.

14. Cirenajwis H, Ekedahl H, Lauss M, Harbst K, Carneiro A, Enoksson J, et al. Molecular stratification of metastatic melanoma using gene expression profiling: Prediction of survival outcome and benefit from molecular targeted therapy. Oncotarget. 2015; 6: 12297-309.

15. Yoshihara K, Shahmoradgoli M, Martinez E, Vegesna R, Kim H, Torres-Garcia $\mathrm{W}$, et al. Inferring tumour purity and stromal and immune cell admixture from expression data. Nat Commun. 2013; 4: 2612.

16. Newman AM, Liu CL, Green MR, Gentles AJ, Feng W, Xu Y, et al. Robust enumeration of cell subsets from tissue expression profiles. Nat Methods. 2015; $12: 453-7$.

17. Robinson MD, McCarthy DJ, Smyth GK. edgeR: a Bioconductor package for differential expression analysis of digital gene expression data. Bioinformatics. 2010; 26: 139-40.

18. Li R, Qu H, Wang S, Wei J, Zhang L, Ma R, et al. GDCRNATools: an $\mathrm{R} /$ Bioconductor package for integrative analysis of IncRNA, miRNA and mRNA data in GDC. Bioinformatics. 2018; 34: 2515-7.

19. Chou $\mathrm{CH}$, Shrestha $\mathrm{S}$, Yang $\mathrm{CD}$, Chang NW, Lin $\mathrm{YL}$, Liao $\mathrm{KW}$, et al. miRTarBase update 2018: a resource for experimentally validated microRNA-target interactions. Nucleic Acids Res. 2018; 46: D296-D302.

20. Paraskevopoulou MD, Vlachos IS, Karagkouni D, Georgakilas G, Kanellos I, Vergoulis $\mathrm{T}$, et al. DIANA-LncBase v2: indexing microRNA targets on non-coding transcripts. Nucleic Acids Res. 2016; 44: D231-8.

21. Shannon P, Markiel A, Ozier O, Baliga NS, Wang JT, Ramage D, et al. Cytoscape: a software environment for integrated models of biomolecular interaction networks. Genome research. 2003; 13: 2498-504.

22. Bindea G, Mlecnik B, Hackl H, Charoentong P, Tosolini M, Kirilovsky A, et al. ClueGO: a Cytoscape plug-in to decipher functionally grouped gene ontology and pathway annotation networks. Bioinformatics. 2009; 25: 1091-3.

23. Kuleshov MV, Jones MR, Rouillard AD, Fernandez NF, Duan Q, Wang Z, et al. Enrichr: a comprehensive gene set enrichment analysis web server 2016 update. Nucleic acids research. 2016; 44: W90-7.

24. Rich JT, Neely JG, Paniello RC, Voelker CC, Nussenbaum B, Wang EW. A practical guide to understanding Kaplan-Meier curves. Otolaryngol Head Neck Surg. 2010; 143: 331-6.

25. Szklarczyk D, Gable AL, Lyon D, Junge A, Wyder S, Huerta-Cepas J, et al. STRING v11: protein-protein association networks with increased coverage, supporting functional discovery in genome-wide experimental datasets. Nucleic Acids Res. 2019; 47: D607-D13.

26. Bader GD, Hogue CW. An automated method for finding molecular complexes in large protein interaction networks. BMC Bioinformatics. 2003; 4: 2.

27. Cancer Genome Atlas N. Genomic Classification of Cutaneous Melanoma. Cell. 2015; 161: 1681-96.

28. Cui T, Zhang L, Huang Y, Yi Y, Tan P, Zhao Y, et al. MNDR v2.0: an updated resource of ncRNA-disease associations in mammals. Nucleic Acids Res. 2018; 46: D371-D4.

29. Schoenborn JR, Wilson CB. Regulation of interferon-gamma during innate and adaptive immune responses. Adv Immunol. 2007; 96: 41-101.

30. Hopkins AL. Network pharmacology: the next paradigm in drug discovery. Nat Chem Biol. 2008; 4: 682-90.

31. Li YH, Yu CY, Li XX, Zhang P, Tang J, Yang Q, et al. Therapeutic target database update 2018: enriched resource for facilitating bench-to-clinic research of targeted therapeutics. Nucleic Acids Res. 2018; 46: D1121-D7.

32. Ramirez AG, Wages NA, Hu Y, Smolkin ME, Slingluff CL, Jr. Defining the effects of age and gender on immune response and outcomes to melanoma vaccination: a retrospective analysis of a single-institution clinical trials' experience. Cancer Immunol Immunother. 2015; 64: 1531-9.

33. Stidham KR, Johnson JL, Seigler HF. Survival superiority of females with melanoma. A multivariate analysis of 6383 patients exploring the significance of gender in prognostic outcome. Arch Surg. 1994; 129: 316-24.

34. Dronca RS, Dong H. A gender factor in shaping T-cell immunity to melanoma. Front Oncol. 2015; 5: 8

35. Thomas NE, Busam KJ, From L, Kricker A, Armstrong BK, Anton-Culver H, et al. Tumor-infiltrating lymphocyte grade in primary melanomas is independently associated with melanoma-specific survival in the population-based genes, environment and melanoma study. J Clin Oncol. 2013; 31: 4252-9.

36. Dabrosin N, Sloth Juul K, Baehr Georgsen I, Andrup S, Schmidt H, Steiniche T, et al. Innate immune cell infiltration in melanoma metastases affects survival and is associated with BRAFV600E mutation status. Melanoma Res. 2019; 29: 30-7.

37. Pozniak J, Nsengimana J, Laye JP, O'Shea SJ, Diaz JMS, Droop AP, et al Genetic and Environmental Determinants of Immune Response to Cutaneous Melanoma. Cancer Res. 2019; 79: 2684-96.

38. Fenimore J, H AY. Regulation of IFN-gamma Expression. Adv Exp Med Biol. 2016; 941: 1-19.

39. Savan R. Post-transcriptional regulation of interferons and their signaling pathways. J Interferon Cytokine Res. 2014; 34: 318-29.

40. Yang L, Li A, Lei Q, Zhang Y. Tumor-intrinsic signaling pathways: key roles in the regulation of the immunosuppressive tumor microenvironment. J Hematol Oncol. 2019; 12: 125
41. Ott PA, Henry T, Baranda SJ, Frleta D, Manches O, Bogunovic D, et al. Inhibition of both BRAF and MEK in BRAF(V600E) mutant melanoma restores compromised dendritic cell (DC) function while having differential direct ffects on DC properties. Cancer Immunol Immunother. 2013; 62: 811-22.

42. Steinberg SM, Shabaneh TB, Zhang P, Martyanov V, Li Z, Malik BT, et al. Myeloid Cells That Impair Immunotherapy Are Restored in Melanomas with Acquired Resistance to BRAF Inhibitors. Cancer Res. 2017; 77: 1599-610. 